wird durch Abdestillieren des Chloroforms erhalten, doch wird man meist vorziehen, letzteres zum Ansatz weiterer Portionen zu gebrauchen. Da $v$. Baeyer keine näheren Angaben macht, sei noch erwähnt, dass sich die Substanz bei $287^{\circ}$ unter Gasentwicklung und Grünfärbung zersetzt. Sie ist löslich in viel Eisessig oder Acetylentetrachlorid und zeigt ihren chinoïden Charakter durch Oxydation von angesäuerter Natriumjodid-Acetonlösung.

Basel, Anstalt für Organische Chemie, Juni 1921.

\title{
Über die Einwirkung von Phenylhydrazin auf Isatogene.
}

(III. Mitteilung ${ }^{1}$ ) über Isatogene)

von

Paul Ruggli und Adolf Bolliger.

(14. VI. 21.)

Anschliessend an unsere Versuche über die Reduktion der isomeren Isatogene haben wir auch Phenylhydrazin auf Isatogene einwirken lassen, da sich dieses Reagens in den Arbeiten von O. Diels und $C$. Salomon ${ }^{2}$ ) bei der Eliminierung des Sauerstoffs aus dem NOCDreiring der cyclischen Anhydride von Dioxy-dihydroglyoxalinen als besonders günstig erwiesen hatte.

Lässt man Phenylhydrazin auf chinoïdes 6-Nitro-2-phenylisatogen (Formel I) einwirken, so wird nicht nur der am Stickstoff stehende Sauerstoff eliminiert, sondern es werden ausserdem zwei Atome Wasserstoff an die Enden des konjugierten Systems addiert, und man erhält 6-Nitro-2-phenylindoxyl (Formel II).<smiles>O=C1C2CCC([N+](=O)[O-])CN2C1c1ccccc1</smiles>

I.<smiles>CCCCCCCCCC1CC2CCC([N+](=O)[O-])CC2N1</smiles>

II.

1) Vergl. die vorstehende Arbeit und B. 52, 1 (1919).

2) B. 52, 45 (1919). 


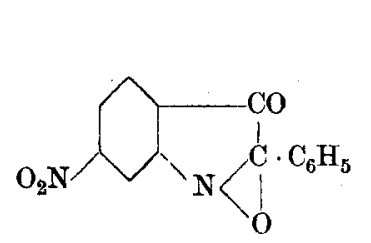

III.<smiles>CCCCNC1CNC2CC([N+](=O)[O-])CCC12</smiles>

IV.

Da die Indoxyle als Hydrochinone der Isatogene aufgefasst werden können ${ }^{1}$ ), verläuft die Reaktion hier analog der Einwirkung von Phenylhydrazin auf gewöhnliches Chinon, wobei bekanntlich Reduktion zu Hydrochinon stattfindet.

Das Nitrophenylindoxyl ist ein sehr beständiger, orangeroter Körper, dessen Farbe sich aus der Anwesenheit von Chromophor $\left(\mathrm{NO}_{2}\right)$ und Auxochrom $(\mathrm{OH})$ erklärt; es wurde durch Darstellung zweier isomerer Acetylderivate von gelber Farbe näher charakterisiert. Als Phenol ist es alkalilöslich und reagiert mit Phenylisocyanat. Das Nitrophenylindoxyl ist unseres Wissens der erste Repräsentant der nitrierten Indoxyle und erscheint uns insofern bemerkenswert, als derartige Körper nach den üblichen Bildungsweisen für Indoxyle nicht ohne weiteres darstellbar sind ${ }^{2}$ ), da die Nitrogruppe in der Regel sowohl gegen Alkalischmelze wie starke Reduktionsmittel empfindlich ist.

Die Hydrochinon- resp. Phenolnatur zeigt sich darin, dass es mit dem chinoïden Nitrophenylisatogen ein typisches Chinhydron bildet. Gibt man die roten Eisessiglösungen der beiden Substanzen zusammen, so krystallisiert beim Erkalten das Chinhydron in tiefbraunschwarzen Nadeln aus. Es ist das beständigste der bisher in dieser Reihe gefundenen Chinhydrone und lässt sich sowohl umkrystallisieren wie auswaschen; in Lösung ist es wie die andern vollständig in seine Komponenten dissoziiert.

Mit der Isoform des Nitrophenylisatogens ${ }^{3}$ ) gibt das Nitrophenylindoxyl keine tieffarbige Substanz.

Lässt man Phenylhydrazin auf die Isoform des Nitrophenylisatogens (III) einwirken, so tritt gleichfalls eine Reaktion ein, und man erhält prachtvolle rote Nadeln, deren Analyse auf ein Phenylhydrazon eines 6-Nitro-2-phenyl-3-keto-indolenins (Formel IV) hin-

1) Vergl. z. B. die Chinhydronbildung, P. Pfeiffer, A. 411, 86 (1916).

$\left.{ }^{2}\right)$ Vergl. z. B. Schwarz, M. 26, 1261 (1905); P. R'uggli, B. 52, 3, 4 (1919).

3) P. Ruggli, B. 52, 5 (1919). 
weist; die Konstitution soll nach Beschaffung von mehr Material durch Spaltung und weitere Reduktion geprüft werden. Die Substanz hat keine oxydierenden Eigenschaften gegen angesäuerte Natriumjodidlösung.

Bringt man Phenylhydrazin in Reaktion mit dem orangefarbenen chinoïden Isatogensäure-methylester (Formel V), so ist der Verlauf ähnlich wiè beim chinoïden Nitrophenylisatogen, doch lässt sich hier noch eine Zwischenstufe isolieren. Geht nämlich die Einwirkung in kalter verdünnter Lösung vor sich, so wird nur der am Stickstoff befindliche Sauerstoff eliminiert, und man erhält das gelbe 2-Carbomethoxy-3-keto-indolenin (Formel VI).

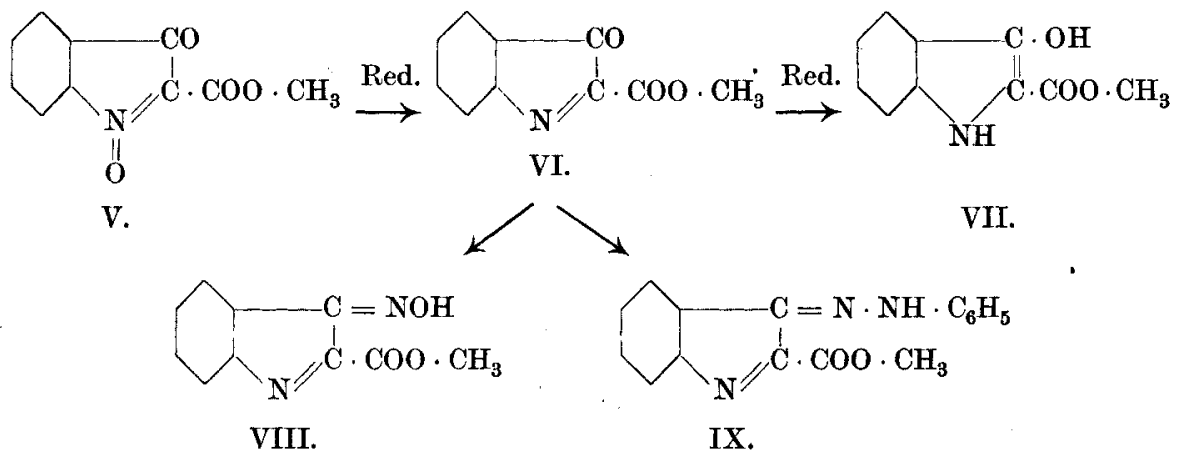

Die hierin noch vorhandene Ketogruppe wurde durch Bildung eines Oxims (Formel VIII) nachgewiesen. Führt man die Einwirkung des Phenylhydrazins in der Wärme weiter, so lagern sich zwei Wasserstoffatome an die konjugierten Doppelbindungen des 2-Carbomethoxy3-keto-indolenins an und es entsteht der bekannte Indoxylsäuremethylester (Formel VII), welcher auch in direkter Reaktion aus Isatogensäure-methylester erhältlich ist. In zwei Fällen erhielten wir bei besonders lebhafter Einwirkung von Phenylhydrazin auf Isatogensäure-methylester eine orange Substanz der Formel $\mathrm{C}_{16} \mathrm{H}_{13} \mathrm{O}_{2} \mathrm{~N}_{3}$, in welcher wahrscheinlich das Phenylhydrazon des 2-Carbomethoxy3-keto-indolenins (Formel IX) vorliegt.

Bei Versuchen, Phenylhydrazin mit Iso-isatogensäure-methylester umzusetzen, haben wir unter den üblichen Bedingungen bis jetzt keine Einwirkung beobachtet. Um indes keinen Zweifel an der Ketogruppe des Isoesters aufkommen zu lassen, haben. wir die Substanz oximiert, worüber in der vorangehenden Mitteilung berichtet wurde. 


\title{
Experimentelles.
}

\author{
6-Nitro-2-phenylindoxyl

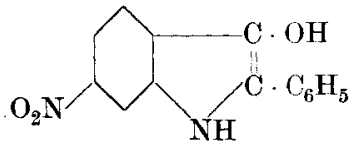

1,5 gr 6-Nitro-2-phenylisatogen werden mit $25 \mathrm{~cm}^{3}$ Alkohol und $1 \mathrm{~cm}^{3}$ Phenylhydrazin oder $1 / 2-3 / 4 \mathrm{~cm}^{3}$ Hydrazinhydrat zusammengegeben und etwa eine halbe Stunde auf dem Wasserbad erwärmt, bis die lebhafte Stickstoffentwicklung vorüber und alles mit tiefroter Farbe in Lösung gegangen ist. Die eventuell filtrierte Lösung wird auf ein Drittel ihres Volumens eingedampit; beim Erkalten erstarrt sie zu einem Krystallbrei orangeroter Blättchen, welche, aus Äthylalkohol umkrystallisiert, bei $215^{0}$ etwas unscharf schmelzen. Die Ausbeute ist sehr gut; die Reinigung ist bei Anwendung von Hydrazin statt Phenylhydrazin etwas leichter. Die Substanz ist in den gebräuchlichen Lösungsmitteln in der Wärme gut löslich, ebenso in kalter Natronlauge, aus welcher sie durch Säuern wieder gefällt wird.

20,970 mgr Subst. gaben 50,850 mgr $\mathrm{CO}_{2}$ und 7,490 mgr $\mathrm{H}_{2} \mathrm{O}$ 21,285 mgr Subst. gaben 51,895 mgr $\mathrm{CO}_{2}$ und $8,015 \mathrm{mgr}_{2} \mathrm{O}$ 0,1608 gr Subst. gaben $16,0 \mathrm{~cm}^{3} \mathrm{~N}_{2}\left(17^{0}, 739 \mathrm{~mm}\right)$.

$$
\begin{array}{llll}
\mathrm{C}_{14} \mathrm{H}_{10} \mathrm{O}_{3} \mathrm{~N}_{2} & \begin{array}{l}
\text { Ber. C } 66 ; 12 \\
\text { Gef. , 66,15; 66,52 }
\end{array} & \text { H } 3,97 & \text { N } 11,00 ; 4,21
\end{array}
$$

\section{Acetylderivate.}

Je nach der Temperatur entstehen zwei verschiedene Acetylverbindungen, welche beide in Natronlauge unlöslich sind; ein N-Acetylderivat liegt also hier nicht vor. Das eine der beiden Isomeren ist jedenfalls das O-Derivat, das andere kann man als C-Derivat auffassen, doch sollen sie in Ermanglung näherer Untersuchungen hierüber einfach als $\alpha$ - und $\beta$-Verbindung bezeichnet werden.

$\alpha$-Acetylverbindung: 0,2 gr 6 -Nitro-2-phenylindoxyl werden mit $12 \mathrm{~cm}^{3}$ Essigsäure-anhydrid, welches eine Spur konzentrierter Schwefelsäure enthält, übergossen. Die Substanz löst sich auf; nach einigen Minuten wird in Wasser gegossen und der orangegelbe Niederschlag wiederholt aus Alkohol umkrystallisiert. Orangegelbe Nadeln vom Smp. $192^{\circ}-193^{\circ}$.

$0,215 \mathrm{I}$ gr Subst. gaben 0,5050 gr $\mathrm{CO}_{2}$ und 0,0859 gr $\mathrm{H}_{2} \mathrm{O}$

$0,1170 \mathrm{gr}$ Subst. gaben $9,9 \mathrm{~cm}^{3} \mathrm{~N}_{2}\left(15^{0}, 739 \mathrm{~mm}\right)$.

$\mathrm{C}_{10} \mathrm{H}_{12} \mathrm{O}_{4} \mathrm{~N}_{2}$ Ber. C 64,26 H 4,05 N 9,46\%

Gef. , $64,04,, 4,37,, 9,58 \%$ 
$\beta$-Acetylverbindung: $1 \mathrm{gr}$ Nitrophenylindoxyl wird mit $7 \mathrm{~cm}^{3}$ Essigsäure-anhydrid und einer Spur Schwefelsäure versetzt; in dieser höheren Konzentration tritt Selbsterwärmung ein, die durch eventuelle Kühlung auf ca. $40^{\circ}$ gehalten wird. Die dunkle Lösung wird in Wasser gegossen und nach Verschwinden des Anhydrids der Niederschlag wiederholt aus Alkohol umkrystallisiert. Grünlich gelbe Nädelchen vom Smp. $172^{\circ}$ (Erweichen bei $164^{\circ}$ ). Mit konzentrierter Schwefelsäure entsteht eine gelbrote Färbung.

20,300 mgr Subst. gaben 48,050 mgr $\mathrm{CO}_{2}$ und 8,195 $\mathrm{mgr} \mathrm{H}_{2} \mathrm{O}$

$10,105 \mathrm{mgr}$ Subst. gaben $0,864 \mathrm{~cm}^{3} \mathrm{~N}_{2}\left(15^{0}, 715 \mathrm{~mm}\right)$.

$$
\begin{aligned}
& \mathrm{C}_{16} \mathrm{H}_{12} \mathrm{O}_{4} \mathrm{~N}_{2} \text { Ber. C 64,26 H 4,05 N 9,46\% } \\
& \text { Gef. ,64,57 , 4,50 ,9,36\% }
\end{aligned}
$$

Die Mischprobe von $\alpha$ - und $\beta$-Acetylverbindung ergibt eine Schmelzpunktsdepression.

Chinhydron aus 6-Nitro-2-phenylisatogen und 6-Nitro-2-phenylindoxyl.

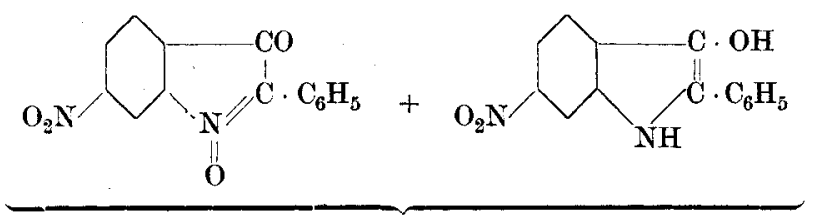

Man gibt gleiche Mengen beider Komponenten, eventuell mit kleinem Überschuss der Indoxylkomponente, in heissem Eisessig gelöst zusammen. Die heisse Mischung zeigt zunächst keine Farbveränderung; beim Erkalten fallen die braunschwarzen Nadeln des Chinhydrons aus, welches aus Eisessig umkrystallisiert wird. Smp. $208^{\circ}$ bis $210^{\circ}$. Es enthält die Komponenten im Verhältnis eins zu eins.

21,390 mgr Subst. gaben 50,510 mgr $\mathrm{CO}_{2}$ und 6,650 mgr $\mathrm{H}_{2} \mathrm{O}$

$11,360 \mathrm{mgr}$ Subst. gaben $1,09 \mathrm{~cm}^{3} \mathrm{~N}_{2}\left(15^{0}, 715 \mathrm{~mm}\right)$

$$
\begin{array}{lllll}
\mathrm{C}_{28} \mathrm{H}_{18} \mathrm{O}_{7} \mathrm{~N}_{4} & \text { Ber. C } 64,23 & \text { H } 3,47 & \mathrm{~N} 10,71 \% \\
& \text { Gef. , 64,42 } & \text {, } 3,48 & \text {, } 10,51 \%
\end{array}
$$

Einwirkung von Phenylhydrazin auf die Isoform des 6-Nitro-2-phenylisatogens.

In kalter verdünnter Lösung tritt keine Reaktion ein. 1,5 gr Iso-nitrophenylisatogen werden mit einer Lösung von $1 \mathrm{~cm}^{3}$ Phenylhydrazin in $24 \mathrm{~cm}^{3}$ Alkohol auf dem Wasserbad gekocht. Nach etwa 20 Minuten beginnt die Substanz sich zu lösen und es tritt Gasentwicklung ein. Zehn Minuten später ist alles mit dunkelrotbrauner Farbe gelöst. Beim Erkalten krystallisieren 0,8 gr eines roten Körpers, 
welcher nach zweimaligem Umkrystallisieren aus viel Alkohol prächtige rote Nadeln vom Smp. $260^{\circ}-261^{\circ}$ bildet. Die Analyse stimmt auf das Phenylhydrazon des 6-Nitro-2-phenyl-3-keto-indolenins (Formel IV).

$20,450 \mathrm{mgr}$ Subst. gaben 52,470 mgr $\left(\mathrm{O}_{2}\right.$ und 8,275 mgr $\mathrm{H}_{2} \mathrm{O}$

$$
\begin{array}{rll}
\mathrm{C}_{20} \mathrm{H}_{14} \mathrm{O}_{2} \mathrm{~N}_{4} & \begin{array}{l}
\text { Ber. C 70,17 } \\
\text { Gef. , 70,00 }
\end{array} & \text { H } 4,09 \% \\
& \text { Gef } 4,52 \%
\end{array}
$$

2-Carbomethoxy-3-keto-indolenin.<smiles>COC(=O)C1=NC2CCCCC2C1=O</smiles>

$4 \mathrm{gr}$ chinoïder Isatogensäure-methylester werden in $80 \mathrm{~cm}^{3}$ Methylalkohol suspendiert; unter Eiskühlung wird eine Lösung von $2 \mathrm{~cm}^{3}$ Phenylhydrazin in $20 \mathrm{~cm}^{3}$ Methylalkohol tropfenweise unter Umschwenken zugegeben. Dann lässt man die Mischung sich auf Zimmertemperatur erwärmen. Ganz allmählich beginnen sich feine Blasen von Stickstoff zu entwickeln, doch darf die Reaktion niemals zu lebhaft werden, da sonst die Reduktion weiter geht. Es beginnt sich nach und nach ein gelbes Pulver neben dem noch ungelösten Isatogensäure-ester abzuscheiden; schliesslith wird die Reaktion durch gelindes Erwärmen auf dem Wasserbad bei einer Innentemperatur von $40^{\circ}-50^{\circ}$ vervollständigt. Nach dem Erkalten wird der gelbe Niederschlag abgesaugt und der Rest aus der Mutterlauge mit sehr verdünnter Salzsäure ausgefällt. Die Ausheute beträgt 3,5 gr. Man krystallisiert aus heissem Nitrobenzol um und setzt vor dem Absaugen. noch ein gleiches Volum Äther zu, worauf die Substanz mit Äther oder Alkohol gut ausgewaschen und auf dem Wasserbad zur Konstanz getrocknet wird. Feine, glänzend gelbe Nädelchen; Smp. unter lebhafter Zersetzung bei ca. $255^{\circ}$ (mässig rasch erhitzt, ab $230^{\circ}$ rotbraune Färbung). Gut löslich in heissem Nitrobenzol, einigermassen auch in heissem Xylol; nicht löslich in Natronlange. Sehr schwer verbrennlich. In konzentrierter Schwefelsäure zunächst gelb, dann olivgrün löslich, beim Erwärmen rot, braun und schliesslich dauernd indigoblau; in der Kälte tritt letztere Färbung erst nach Wochen auf.

21,300 mgr Subst. gaben 49,330 mgr $\mathrm{CO}_{2}$ und 8,015 $\mathrm{mgr} \mathrm{H}_{2} \mathrm{O}$

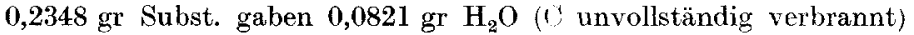

8,609 mgr Subst. gaben $0,36 \mathrm{~cm}^{3} \mathrm{~N}_{2}\left(25^{0}, 715\right)$

0,1749 gr Subst. gaben $11,7 \mathrm{~cm}^{3} \mathrm{~N}_{2}\left(20^{4}, 741 \mathrm{~mm}\right)$

$$
\begin{array}{llll}
\mathrm{C}_{10} \mathrm{H}_{7} \mathrm{O}_{3} \mathrm{~N} & \text { Ber. C } 63,48 & \mathrm{H} 3,73 & \mathrm{~N} \mathrm{7,40 \%} \\
& \text { Gef. , 63,18 } & \text {,4,21;3,91 } & \text {, 7,57; 7,42\% }
\end{array}
$$


Oxim. 1 gr 2-Carbomethoxy-3-keto-indolenin und 3,5 gr Hydroxylaminchlorhydrat werden in $40 \mathrm{~cm}^{3}$ Alkohol etwa eine halbe Stunde auf dem Wasserbad bis zur völligen Lösung erwärmt, eventuell filtriert und über Nacht stehen gelassen. Es krystallisiert das Oxim vorläufig grünstichig gelb, nur etwa $0,4 \mathrm{gr}$; die Mutterlauge enthält amorphe grüne Substanzen. Nach Umkrystallisieren aus Alkohol besteht das Oxim ans rein gelben Nädelchen vom Smp. $221^{0}$ (Zersetzung).

$$
\begin{aligned}
& 11,960 \mathrm{mgr} \text { Subst. gaben } 1,55 \mathrm{~cm}^{3} \mathrm{~N}_{2}\left(23^{0}, 711 \mathrm{~mm}\right) \\
& \mathrm{C}_{10} \mathrm{H}_{8} \mathrm{O}_{3} \mathrm{~N}_{2} \text { Ber. } \mathrm{N} 13,72 \text { Gef. } \mathrm{N} 13,98 \%
\end{aligned}
$$

\section{Indoxylsäure-methylester $\left.{ }^{1}\right)$.}

(Formel VII.)

a) Durch Reduktion von 2-Carbomethoxy-3-keto-indolenin (Formel $\mathrm{VI} \rightarrow$ VII) : $0,9 \mathrm{gr}$ 2-Carbomethoxy-3-keto-indolenin werden mit $1,2 \mathrm{~cm}^{3}$ -Phenylhydrazin in $20 \mathrm{~cm}^{3}$ Alkohol auf dem Wasserbad etwa 45 Minuten unter Rückfluss gekocht, bis alles gelöst ist. Aus der Lösung krystallisieren nach dem Eindampfen beim Erkalten die Nadeln des Indoxylsäure-methylesters aus. Sie wurden durch Analyse, den Smp. 156 und die Mischprobe mit einem analog der Methode von Vorländer und v. Schilling ${ }^{2}$ ) gewonnenen Präparat identifiziert.

20,190 mgr Subst. gaben 46,800 mgr $\mathrm{CO}_{2}$ und $8,510 \mathrm{mgr} \mathrm{H}_{2} \mathrm{O}$ 10,000 mgr Subst. gaben $0,65 \mathrm{~cm}^{3} \mathrm{~N}_{2}\left(14^{0}, 713 \mathrm{~mm}\right)$.

$$
\begin{array}{rccc}
\mathrm{C}_{10} \mathrm{H}_{9} \mathrm{O}_{3} \mathrm{~N} & \text { Ber. C 62,82 } & \text { H 4,71 } & \mathrm{N} 7,33 \% \\
& \text { Gef. , } 63,23 & , 4,72 & , 7,13 \%
\end{array}
$$

b) Aus Isatogensäure-methylester und Phenylhydrazin (Formel $\mathrm{V} \rightarrow \mathrm{VII}): 2,5 \mathrm{gr}$ chinoïder Isatogensäure-methylester werden in $25 \mathrm{~cm}^{3}$ Alkohol suspendiert und $2,5 \mathrm{~cm}^{3}$ Phenylhydrazin zugegeben. Es tritt spontan eine heftige Reaktion ein, welche man durch etwa drei Minuten langes Erwärmen auf dem Wasserbad vervollständigt. Beim Erkalten krystallisiert der Indoxylsäure-methylester zunächst mehr oder weniger rotbraun gefärbt aus. Er wird durch Behandlung mit Tierkohle entfärbt und zeigt dann den Smp. $156^{\circ}$; er wurde durch Mischprobe identifiziert.

1) Vorländer und $v$. Schilling, A. 301, 351 (1898).

2) A. 301, 350 (1898). 
Phenylhydrazon des 2-Carbomethoxy-3-keto-indolenins.

(Formel IX ?)

In zwei Fällen erhielten wir bei der Einwirkung von Phenylhydrazin auf Isatogensäure-methylester, wie sie in dem eben genannten Versuch zum Indoxylsäure-ester führte, orange Krystalle, welche zweimal aus Alkohol umkrystallisiert bei $218^{\circ}$ schmolzen. Sie zeigten kein Oxydationsvermögen gegen angesäuerte Natriumjodid-Acetonlösung.

21,090 mgr Subst. gaben 53,515 mgr $\mathrm{CO}_{2}$ und $9,020 \mathrm{mgr} \mathrm{H}_{2} \mathrm{O}$ 9,985 mgr Subst. gaben $1,36 \mathrm{~cm}^{3} \mathrm{~N}_{2}\left(15^{0}, 723 \mathrm{~mm}\right)$

$$
\begin{array}{llllll}
\mathrm{C}_{16} \mathrm{H}_{13} \mathrm{O}_{2} \mathrm{~N}_{9} & \text { Ber. C } 68,82 & \mathrm{H} & 4,66 & \mathrm{~N} & 15,05 \% \\
& \text { Gef. , } 69,21 & , 4,78 & , .15,11 \%
\end{array}
$$

Basel, Anstalt für organische Chemie, Juni 1921.

\title{
De l'action de l'ortho-aminothiophénol sur les orthoquinones III
}

\author{
par \\ Knut Stahrfoss.
}

(15. VI. 21.)

La réaction entre l'o-aminothiophénol et la 4 -amino- $\beta$-naphtoquinone dans l'acide acétique donne lieu à la formation de plusieurs substances, dont deux ont été décrites dans des publications précédentes $\left.^{1}\right)$ :

I.<smiles>N=C1CC2CC3CCCCC3CC2C2=C1CCCCC2</smiles>

naphto-phénothiazime-6

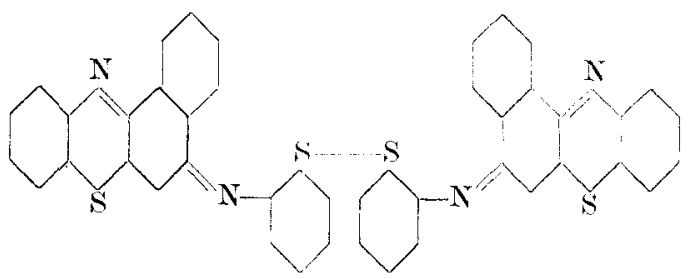

disulfure de l'o-mercaptophényl-naphto-

phénothiazime-6

II.

1) Helv. 3, 134 (1920); Helv. 4, 273 (1921). 